\title{
Nonlinear photonic switching by using the spatial soliton collision
}

\author{
Tian-Tsorng Shi and Sien Chi \\ Institute of Electro-Optical Engineering, National Chiao Tung University, Hsinchu, Taiwan, China
}

Received February 26, 1990; accepted July 13, 1990

\begin{abstract}
We introduce a novel device for optical switching by using the position shift of the spatial soliton after collision. The device is a one-to-three switching device controlled by two control beams. For the optimum signal power, the transmission efficiency is above $95 \%$. The wave propagation in the structure and the transmission efficiency as a function of input signal power are shown and discussed.
\end{abstract}

All-optical switching devices have the potential for applications in signal processing and telecommunications. In recent years, much research has been carried out in optical switching. ${ }^{1-4}$ In the past decade much attention has been focused on the nonlinear directional coupler, ${ }^{5,6}$ in which the light is switched by controlling its intensity. Owing to the instability in the nonlinear directional coupler, one can control the switching behavior with a weak control signal. ${ }^{7}$ Recently a switch was designed by using soliton collisions in waveguides. ${ }^{8}$ Here we propose a different method to achieve optical switching by using the position shift of the spatial soliton after collision. For the optimum signal power, the transmission efficiency is above $95 \%$.

The structure of the proposed optical switching device is shown in Fig. 1. This device is a one-to-three switching device controlled by two control light beams. The structure of the device is divided into three sections: an input section, a nonlinear medium section, and an output section. In the input section the signal guide is in the center and the two outside guides are control guides. The two control guides are inclined toward the signal guide so that the control beam and the signal beam can collide in the nonlinear medium. The separation between each guide in the input section is sufficiently large to prevent mutual coupling. A nonlinear medium is placed between the input section and the output section. ${ }^{9}$ In this medium the spatial solitons are excited and collide with each other. ${ }^{10}$ The third section, the output section, has five guides. The outer two, called control guides, are used to couple out the control beams. If the control beam is useless in the following process, we may use a lossy medium to attenuate it. The middle three guides, called signal guides, are used to couple out the spatial soliton excited by the input signal. The separation between the outside signal guide and the center guide is designed to be equal to the amount of position shift due to soliton collision. Each soliton excited by the signal will be trapped by one of the three output signal guides whether or not it collides with the control beam. Because the separation between each guide is small, there exist strong couplings between the guides. From the studies of the three-guide optical coupler, ${ }^{11}$ we can choose an optimum coupling length to single out the signal from one of the three guides. Therefore the two outside signal guides are first parallel to, and then bend out from, the straight center guide.

In order to calculate the light-wave propagation in the structure, the electric field of the light is written as

$$
E(x, z, t)=\mathscr{E}(x, z) \exp \left(i \beta k_{0} z-\omega t\right),
$$

where $k_{0}=2 \pi / \lambda_{0}$ is the wave number in free space, $\beta$ is the effective refractive index, and $\lambda_{0}$ is the wavelength in free space. Since we consider the two-dimensional problem only, the field is assumed to be $y$ independent. By using the slowly varied amplitude approximation, the wave equation is reduced to

$$
-2 i \beta k_{0} \frac{\partial \mathscr{E}}{\partial z}+\frac{\partial^{2} \mathscr{E}}{\partial x^{2}}+k_{0}{ }^{2}\left[n^{2}\left(x, z,|\mathscr{E}|^{2}\right)-\beta^{2}\right] \mathscr{E}=0,
$$

where $n=n_{c}$ in the cladding of the input and output sections, $n=n_{f}$ in the core of the input and output

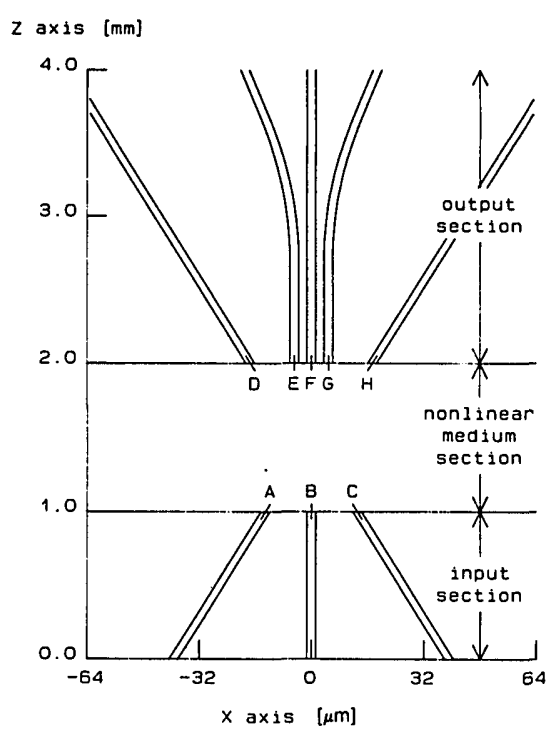

Fig. 1. Structure of the nonlinear photonic switching device. $A, C, D$, and $H$ are control guides; $B, E, F$, and $G$ are signal guides. 


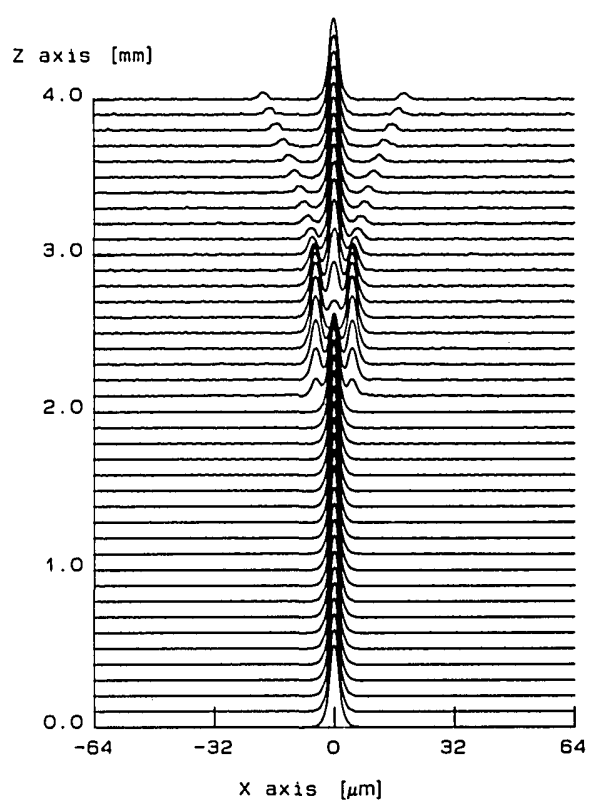

Fig. 2. Propagation of the signal field $|E|$ without the control beam.

sections, and $n=n_{0}+n_{2 I}|\mathscr{E}|^{2}$ in the nonlinear medium section.

We use the beam-propagation method ${ }^{12}$ to simulate the propagation phenomena of the light beam in the structure. For the numerical calculation we use the following data: the wavelength of the light in free space $\lambda_{0}=1.3 \mu \mathrm{m}$, the refractive index of the cladding $n_{c}=1.55$, the refractive index of the core $n_{f}=1.57$, the width of the waveguide core is $2.5 \mu \mathrm{m}, n_{0}=1.55$ and $n_{2 I}$ $=5.3 \times 10^{-10} \mathrm{~m}^{2} / \mathrm{W}$ in the nonlinear medium, ${ }^{8}$ the input signal or control beam is chosen as the eigenmode of the waveguide, the power of control beam is fixed at $P_{0}=0.214 \mathrm{~W} / \mathrm{mm}$, and the power of the signal is varied from $0.4 P_{0}$ to $1.4 P_{0}$.

When there is no control beam, the propagation of the signal beam in the structure is shown in Fig. 2. An eigenmode of the signal guide is launched. The wave first propagates in the input section $(z=0$ to $1 \mathrm{~mm})$ unchanged, and then it meets the nonlinear medium. It excites the spatial soliton with a similar shape as the eigenmode of the signal guide. The reflection of the interface is neglected. Because of the small thickness of the nonlinear medium $(1 \mathrm{~mm})$, we neglect the scattering and absorption losses, and the spatial soliton propagates unchangedly in the medium. Then the spatial soliton is coupled out by the middle signal guide in the output section. After the mutual coupling process, the signal beam is separated from the other two outside signal guides. The transmission efficiency in this condition is greater than $97 \%$.

When the control beam is on, the switching behavior is shown in Fig. 3. The signal is incident from the center signal guide, and the control beam is incident from the left-hand-side control guide. The power of the signal beam is assumed to be equal to that of the control beam. The two beams propagate along the guides in the input section and excite the spatial solitons in the nonlinear medium. Because the two guides are not parallel, the two excited spatial solitons collide in the nonlinear medium. After the collision, except for the transverse position shift, both the amplitude and the propagation direction of each spatial soliton are unchanged. In the output section, the control beam is coupled out by the right-hand-side output control guide, and the signal is coupled out by the lefthand-side signal guide. After a half-period of mutual coupling, the signal is transferred to the right-handside output signal guide and is separated out. Owing to the symmetry, when the control beam comes from the right-hand-side input control guide, the signal will be switched to the left-hand-side output signal guide.

Because the refractive index of the nonlinear medi$u m$ is intensity dependent, the transmission efficiency

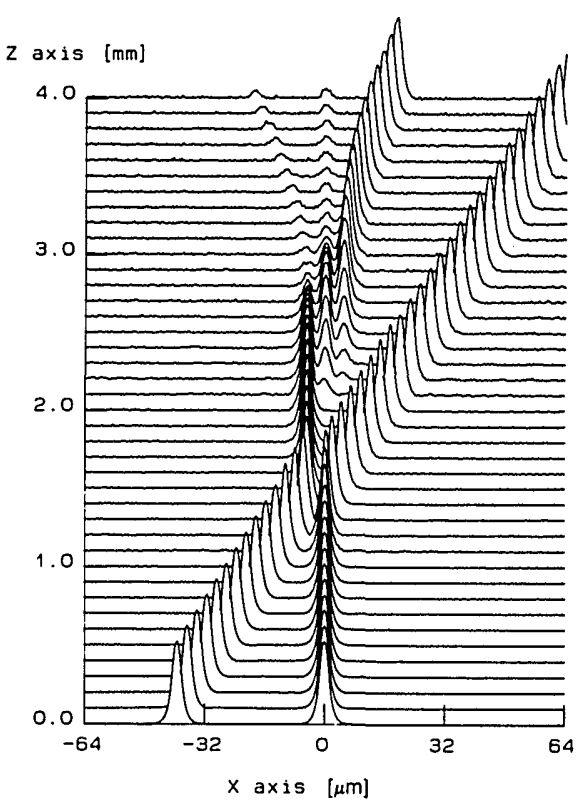

Fig. 3. Propagation of the signal field $|E|$ with the left control beam.

$$
\begin{aligned}
& \text { signal power } \\
& \text { output/input }
\end{aligned}
$$

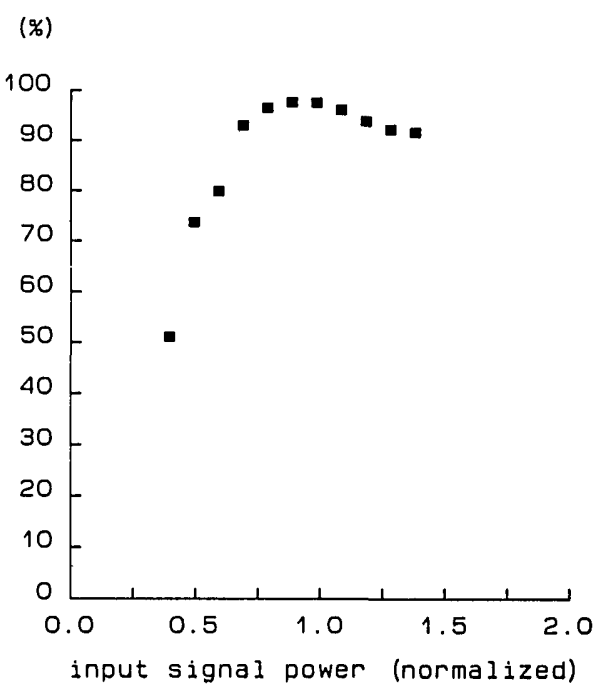

Fig. 4. Transmission efficiency as a function of the input signal power (normalized to the optimum signal power) in the case of no control beam. 
signal power

output/input

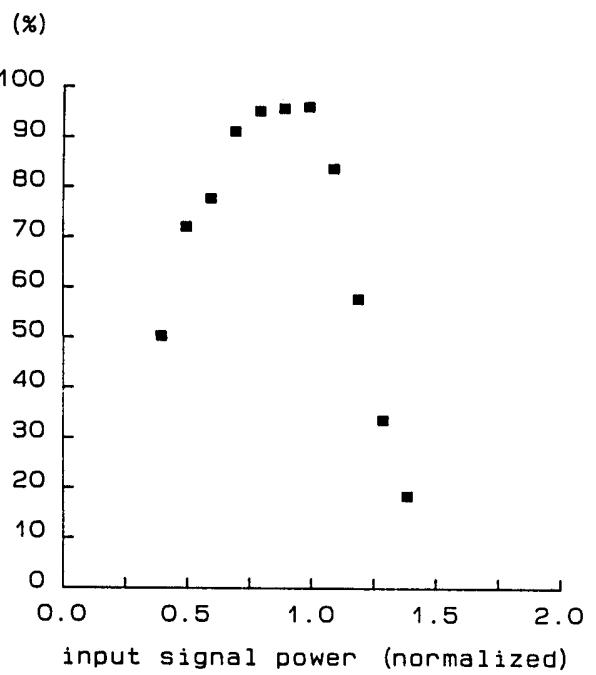

Fig. 5. Transmission efficiency as a function of the input signal power when the control beam is on.

of this device will be dependent on the intensity of the input signal. We define the input signal power as the optimum signal power when the transmission efficiency reaches maximum. Figure 4 shows the transmission efficiency as a function of input signal power without the control beam. When the signal beam is different from the optimum power, the excited spatial soliton will not be a fundamental one again; there is a mismatch between the shape of the spatial soliton and the eigenmode of the output signal guide. Thus when the spatial soliton is coupled out in the output signal guide, there will be some radiation loss, which makes the transmission efficiency lower than optimum. Figure 5 shows the transmission efficiency as function of input signal power with the control beam. The control beam power is assumed to be equal to the optimum signal power. When the input signal power is lower than the optimum signal power, the plot is similar to that in Fig. 4. When the input signal power is higher than the optimum signal power, the position shift of the signal spatial soliton is less. Part of the signal power falls in the cladding area between the center output signal guide and the outside output signal guide; hence there is a great radiation loss. Therefore the transmission efficiency drops drastically when the input signal power is higher than the optimum power.

In conclusion, we have introduced a new optical switching device by using the characteristics of spatial soliton collision. The proposed structure is a one-tothree switching device, controlled by two control beams. When the control beam comes from the righthand-side, the signal will be switched to the left-handside output signal guide, and vice versa. If there is no control beam, the signal will stay in the center signal guide. Since one collision causes a certain amount of position shift, and two collisions will cause a double position shift, we can use this shift to make 1-to- $N$ switching devices. This device has potential applications in optical signal processing and telecommunications.

\section{References}

1. T. K. Gustafson and P. W. Smith, eds., Photonic Switching (Springer-Verlag, Berlin, 1988).

2. J. P. Sabini, N. Finlayson, and G. I. Stegeman, Appl. Phys. Lett. 55, 1176 (1989).

3. Y. Silberberg and B. G. Sfez, Opt. Lett. 13, 1132 (1988).

4. D. R. Heatley, E. M. Wright, and G. I. Stegeman, Appl. Phys. Lett. 53, 172 (1988).

5. S. M. Jensen, IEEE J. Quantum Electron. QE-18, 1580 (1982).

6. A. Ankiewicz, Opt. Quantum Electron. 20, 329 (1988).

7. S. Wabnitz, E. M. Wright, C. T. Seaton, and G. I. Stegeman, Appl. Phys. Lett. 49, 838 (1986).

8. A. B. Aceves, J. V. Moloney, and A. C. Newell, Phys. Rev. A 39, 1828 (1989).

9. S. Chi and T. T. Shi, Electron. Lett. 26, 339 (1990).

10. S. Chi and S. Wen, Opt. Commun. 69, 334 (1989).

11. J. P. Donnelly, H. A. Haus, and N. Whitaker, IEEE J. Quantum Electron. QE-23, 401 (1987).

12. M. D. Feit and J. A. Fleck, Jr., Appl. Opt. 19, 1154 (1980). 